\title{
THEORY COMPRESSION WITH ELLIPTIC FUNCTIONS
}

\author{
VICTOR A. BRUMBERG \\ Bureau des Longitudes \\ 77, av. Denfert-Rochereau, Paris 75014, France \\ (On leave from Institute of Applied Astronomy \\ 8, Zhdanovskaya st., St.-Petersburg 197042, Russia)
}

\begin{abstract}
Introduction of Jacobi elliptic functions in planetary, satellite and cometary problems of celestial mechanics is a transformation of variables to present the analytical theories of motion in the more compact form as compared with the traditional series in multiples of mean longitudes or mean anomalies.
\end{abstract}

\section{Introduction}

Analytical techniques of celestial mechanics survive now not the best period in their history. In relation to the two-century anniversary of Bureau des Longitudes, the world-recognized center of analytical celestial mechanics, it may be reasonable to discuss once again the role of analytical techniques in celestial mechanics. It is true that numerical integration represents now the best tool to investigate the empirical evolution of dynamical systems and to produce the high accuracy ephemerides of specific bodies. But analytical theories enable one to get a more profound insight into physical and mathematical laws of motion. Indeed, analytical techniques of celestial mechanics are aimed

- to obtain a general solution of the equations of motion as explicit function of initial values and parameters;

- to present a solution in the physically adequate form;

- to be used as a framework to investigate small effects due to extra additive forces in the right-hand members of the equations of motion. 
It should be added that analytical techniques of celestial mechanics contributed a lot into mathematical and natural sciences in such domains as special functions, perturbation theory, stability and resonance theory, periodic solutions, algebraic systems, etc.

It is of interest to compare the role of numerical integration and analytical approach in typical problems of celestial mechanics.

\section{Two-body problem}

It is hardly possible to imagine it without the analytical solution in spite of the fact that the closed-form time-explicit solution of this problem has been actually obtained only quite recently by Osácar and Palacián (1994) with the use of dilogarithmic function. This is an example where analytical treatment due to its simplicity and compactness is far beyond numerical integration approach.

2. Lunar theory

The most recent semi-analytical theory of the motion of the Moon is ELP by Chapront and Chapront-Touzé (1995). This theory is used in Connaissance des Temps but as compared with numerical integration its accuracy is not completely sufficient to analyze the high-precision LLR observations. Nevertheless, by its domain of applicability beyond the scope of LLR this theory significantly surpasses numerical lunar ephemerides.

3. Planetary theories

The semi-analytical theories VSOP of the motion of the major planets by Bretagnon (1982) are used in Connaissance des Temps and in space research as well as numerical planetary ephemerides. Along with this they are of great benefit for astrometry, IERS activity and Earth's sciences.

4. Satellite theory

It is evident that for the analysis of high-precision SLR observations one needs numerical ephemerides. But for a large class of research problems a simple first-order analytical theory may be quite adequate. Kaula's theory is just an example of such wide-purpose analytical theory.

5. Cometary motion

In spite of the efforts of Hansen, Gylden and their followers (see below) this problem remains to be a challenge for analytical techniques and the cometary orbits are computed mostly by numerical integration.

6. Rotation of celestial bodies

Analytical solution of this problem like recent theory of precession and nutation by Kinoshita and Souchay (1990) is quite competent with numerical integration by its accuracy but is much more informative 
in respect of physical interpretation of different components of the solution.

7. GTM (general theory of translatory-rotational motion)

At present, most analytical theories representing translatory or rotational motion of celestial bodies are constructed for the sake of compactness with fictitious secular terms. The physically adequate form of the solution implies that the angular variables are expressed as linear functions of time and the action variables are represented by slowly changing quasi-periodic functions of time. Such theory for the solar system bodies may be called GTM. Its compactness may be achieved by introducing an adequate intermediary (Hill-like type for the translatory motion), separating fast and slow variables in the sense of Zeipel and performing a normalizing (Birkhoff-like) transformation to a secular system. Such a problem is still awaiting its practical solution.

Twenty or thirty years ago it seemed that the desired accuracy of analytical theories could be always achieved by using very long Poisson series constructed by means of Poisson series processors. Now it became evident that the increase of the number of terms in Poisson series cannot be too efficient tool for this aim. The main recipe nowadays is to develop compact-form analytical theories with the aid of more sophisticated specialized software based on some universal computer algebra system (Maple, Mathematica, etc.). A compact-form analytical theory may be understood as a theory with the large value of the ratio of the achieved accuracy to the needed number of terms (like the commercial quality to price ratio). It is evident that the vast arsenal of compression transformations of classical celestial mechanics may be used now in combination with present computer software facilities.

\section{Compact-Form Series in Classical Celestial Mechanics}

Many analytical techniques of classical perturbation theory are based on the two-body problem Fourier expansions in multiples of mean anomaly $M$, true anomaly $v$ or eccentric anomaly $g$

$$
\begin{aligned}
\left(\frac{r}{a}\right)^{n} \operatorname{expi} m v & =\sum_{s=-\infty}^{\infty} X_{s}^{n, m}(e) \operatorname{expi} s M \\
& =\sum_{s=-\infty}^{\infty} Y_{s}^{n, m}(e) \operatorname{expi} s v \\
& =\sum_{s=-\infty}^{\infty} Z_{s}^{n, m}(e) \operatorname{expi} s g
\end{aligned}
$$


Expansion (2.1) enables one to represent the coordinates as explicit functions of time. But Hansen coefficients $X_{s}^{n, m}(e)$ for moderate and large values of eccentricity $e$ decrease not so fast with the increase of $|s|$ so that this expansion may be too long. Expansions (2.2) and (2.3) are more compact in this respect (moreover, they reduce to finite trigonometric polynomials for $n<0$ and $n>m>0$, respectively). In classical celestial mechanics the eccentric anomaly series based on (2.3) were used in particular by Hansen, Newcomb and Hill. Later on the true anomaly series based on (2.2) were used by Brown and Shook (1933). From time to time expansions (2.2) and (2.3) may be met in contemporary papers. But it seems that most people share the negative opinion by Zeipel. In his encyclopaedic paper Zeipel (1912) comes to conclusion that the advantages of the compact representation of the disturbing function based on the eccentric anomaly series might be lost during the integration by means of the transformation to the mean anomalies with the aid of Bessel functions. We shall return below to the key problem of integration with different trigonometric arguments.

The idea to use elliptic functions to find more efficient expansions than (2.1)-(2.3) for compression of analytical theories of celestial mechanics was suggested by Gylden more than a century ago. His idea is that 'one views the mean (true, eccentric) anomaly ... as the elliptic amplitude of a new (independent) variable' (see Nacozy, 1977). Unfortunately, this idea was related to the Hansen method of partial anomalies for the analytical representation of cometary motion. This method involves the division of a cometary orbit into several (at least two) parts with its own independent argument for each part. In spite of all efforts of Gylden and his followers the partial anomaly technique remained and still remains rather cumbersome for wide application (Nacozy, 1969; Skripnichenko, 1972). Since then the idea of Gylden was regarded only as an attempt to improve the partial anomaly technique and numerical integration became the most widespread tool for investigation of the cometary motion.

\section{Elliptic Function Expansions as Convergence Accelerators}

In realizing the Gylden's idea one has not to deal with problems admitting a solution in terms of elliptic functions (as the problem of two fixed centres, for example). Moreover, one has to do not with elliptic functions themselves but rather with their Fourier expansions. The main idea is to find, if possible, a transformation of variables

$$
(x, y) \rightarrow(k, u)
$$

reducing a function $f(x, y)$ of one power $(0 \leq x \leq 1)$ and one trigonometric variable $(0 \leq y \leq 2 \pi)$ to Jacobi elliptic function $g(k, u)$ with modulus $k$ and 
argument $u$. If such transformation (3.1) exists then the ordinary Fourier expansion

$$
f(x, y)=\sum f_{m}(x) \operatorname{expimy}
$$

of function $f(x, y)$ will be replaced by the Fourier expansion

$$
g(k, u)=\sum g_{m}(q) \operatorname{expimw}, \quad w=\frac{\pi u}{2 K(k)}
$$

of function $g(k, u)$. Here $q$ is Jacobi nome remaining comparatively small even for the values of $k$ close to $1 . K(k)$ is the complete elliptic integral of the first kind. For large $|m|$ coefficients $g_{m}$ decrease generally much faster than coefficients $f_{m}$ and one may expect that series (3.3) will be much more compact than series (3.2).

One should underline two points frequently overlooked, i.e.

- transformation (3.1) is made in function $f(x, y)$ but not in series (3.2) which is not needed at all;

- compactness of series (3.3) with respect to (3.2) is due mainly to the $k$-dependent angular variable $w$ and in lesser extent due to the compactness of coefficients $g_{m}$ themselves. Coefficients $f_{m}$ may be computed without power series expansions (Laplace coefficients in planetary problems or Hansen coefficients in satellite problems) or may be represented by closed form expressions (coefficients of (2.2) and (2.3) series) but this has nothing to do with the slow convergence of series (3.2) itself.

The standard Fourier series for simple combinations of Jacobi elliptic functions may be found in many textbooks. They are collected as (2.5.66)(2.5.78) in (Brumberg, 1995). The Fourier series for any rational function of Jacobi elliptic functions may be derived by recurrence relations starting with these standard expansions.

As it is well known even more fast converging expansions are provided by theta functions. But in using these expansions one meets two difficulties, i.e.

- the absence of such expansion for periodic part of elliptic amplitude needed for the inversion and interrelation problem;

- the necessity to perform operations on rational functions of Poisson series.

The latter difficulty occurs also in applying the Landen transformation $(k, u) \rightarrow\left(k_{1}, v\right)$

$$
k=\frac{2 k_{1}^{1 / 2}}{1+k_{1}}, \quad u=\left(1+k_{1}\right) v
$$


enabling one to operate with the Jacobi nome $q_{1}=q^{2}$.

Application of the standard expansions for Jacobi elliptic functions demands a simpler software to perform symbolic operations on trigonometric series with rational coefficients.

\section{Elliptic Functions in Planetary Problems}

The starting point of many analytical investigations in planetary problems is the expansion of a generating function

$$
\gamma(n, x, y, \nu, \alpha, \zeta)=\alpha^{n}\left(1-\alpha \zeta^{-1}\right)^{x}(1-\alpha \zeta)^{y}(-\zeta)^{\nu}
$$

occurring in the right-hand members of the equations of motion. Here $\alpha$ is a real parameter (the ratio of the semi-major axes), $\zeta$ is an exponential function of the mean longitudes $\lambda$ and $\lambda^{\prime}$

$$
\zeta=\exp \mathrm{i}\left(\lambda-\lambda^{\prime}\right)
$$

$n$ and $\nu$ are integers, $x$ and $y$ are real numbers or more precisely

$$
x=-\frac{K}{2}, \quad y=-\frac{L}{2}
$$

where $K$ and $L$ are positive odd integers. Traditional Fourier expansion of function (4.1) has the form

$$
\gamma(n, x, y, \nu, \alpha, \zeta)=\sum_{\sigma=-\infty}^{\infty} \tilde{\gamma}_{\sigma}(n, x, y, \nu, \alpha) \zeta^{\sigma} .
$$

Functions of type (4.1) and their expansions (4.4) were considered by Newcomb, Cauchy and Gylden (see Zeipel, 1912). Later on this function was studied by Brown and Shook (1933) and was intensively used in constructing GPT, general planetary theory (Brumberg, 1995). Quite recently it was applied by Laskar and Robutel (1995) to derive a new expansion of the planetary disturbing function. The main difficulty in applying (4.4) is the slow convergence of this expansion especially for large values of $\alpha$. The possible remedy is to find transformation (3.1) of variables $\left(\alpha, \lambda-\lambda^{\prime}\right)$ to elliptic variables $(k, u)$. Indeed, the transformation

$$
2 \text { am } u=M, \quad M=\pi-\left(\lambda-\lambda^{\prime}\right)
$$

and

$$
k^{2}=\frac{4 \alpha}{(1+\alpha)^{2}}
$$


reduces function (4.1) to the form

$$
\gamma(n, x, y, \nu, \alpha, \zeta)=\frac{\alpha^{n}(-\zeta)^{\nu}}{[(1+\alpha) \operatorname{dn} u]^{\max \{K, L\}}}\left(1-\alpha \zeta^{\operatorname{sgn}(K-L)}\right)^{\frac{|K-L|}{2}}
$$

with

$$
\zeta=-\exp (-\mathrm{i} 2 \text { am } u)
$$

It is easy to see that function (4.7) may be expanded in Fourier series in multiples of

$$
w=\frac{\pi u}{K(k)}
$$

or in the exponential form

$$
\gamma(n, x, y, \nu, \alpha, \zeta)=\sum_{\sigma=-\infty}^{\infty} \gamma_{\sigma}(n, x, y, \nu, \alpha) \tau^{\sigma}, \quad \tau=\operatorname{expi} w
$$

The difference in the arguments (3.3) and (4.9) is due to the fact that the real periods of function (4.7) and of general elliptic function (3.3) are $2 K(k)$ and $4 K(k)$, respectively. Coefficients $\gamma_{\sigma}$ of (4.10) may be easily found in the closed form with respect to Jacobi nome $q$. Indeed, these coefficients are expressed in terms of auxiliary functions $I_{2 m, n}$ defined by

$$
I_{p, n}(u)=\frac{\exp (\mathrm{i} p \operatorname{am} u)}{(\operatorname{dn} u)^{n}}
$$

and for $p=2 m$ (integer $m$ ) these functions are easily expanded in $\tau$-series by means of the recurrence relations (see, for example, Howland, 1988). The most difficult case in the planetary problems is provided by the pair Venus-Earth. For this pair one has $\alpha=0.723, k=0.987$ and $q=0.215$. Even for this pair the $\tau$-expansion (4.10) of generating function (4.1) is much more compact than traditional $\zeta$-series (4.4).

Relationship with time is realized with the aid of (4.5). This relation represents the Lagrange implicit function equation of the form

$$
w+\sum_{m=1}^{\infty} d_{m}(q) \sin m w=M
$$

with

$$
d_{m}(q)=\frac{4}{m} \frac{q^{m}}{1+q^{2 m}} .
$$

The inversion of this equation has the form

$$
w=M+\sum_{m=1}^{\infty} c_{m}(q) \sin m M .
$$


Moreover, for any real $s$ (E.Brumberg et al., 1995)

$$
\exp \mathrm{i} s w=\sum_{r=-\infty}^{\infty} E_{r}^{(s)}(q) \operatorname{expi}(s+r) M
$$

and

$$
\exp \mathrm{i} s M=\sum_{r=-\infty}^{\infty} F_{r}^{(s)}(q) \exp \mathrm{i}(s+r) w .
$$

Recent results by E.Brumberg (1995) for the analogous relations for high eccentricity orbits show that coefficients $F_{r}^{(s)}$ decrease with large $|r|$ much faster than coefficients $E_{r}^{(s)}$.

Transformation (4.5) and (4.6) known already in classical celestial mechanics was applied by Richardson (1982) in his research on planetary intermediate orbits constructed by Lie transforms. Later on the same transformation has been used by Williams et al. (1987) in the attempt to represent the first-order classical planetary theories in $k, u$ variables. At the same time Chapront and Simon (1988) have developed a first-order CPT (compact planetary theory). Replacing mean longitudes $\lambda$ and $\lambda^{\prime}$ by linear function of time $l$ and elliptic argument $u$

$$
\lambda=l-\operatorname{am} u+\frac{\pi}{2}, \quad \lambda^{\prime}=l+\operatorname{am} u-\frac{\pi}{2}
$$

they have compared the traditional perturbation theory series

$$
x=\sum_{k=0}^{m}\left[\tilde{x}_{k}+\tilde{S}_{k}\left(\lambda, \lambda^{\prime}\right)\right] t^{k}
$$

with the elliptic argument series

$$
x=\sum_{k=0}^{m}\left[x_{k}+S_{k}(l, w)\right] w^{k} .
$$

It turned out that series $S_{k}(l, w)$ are much shorter than series $\tilde{S}_{k}\left(\lambda, \lambda^{\prime}\right)$ for all couples of the major planets. Series (4.18) and (4.19) were computed in the semi-analytical form by application of FFT (fast Fourier transform) to the equations of motion. This work is still awaiting its completion.

Transformation (4.5) and (4.6) has been intensively used in elaborating GPT, a planetary theory without fictitious secular terms (Brumberg, 1995). GPT is constructed by the series in powers of the eccentricity and inclination variables with the coefficients depending on the differences of the mean longitudes of the planets. These series reduce the original equations of motion to an autonomous secular system. In dependence on the representation of the GPT series one may distinguish three forms of GPT, i.e. 
(a) $\zeta$-series form as (4.4) extended for the $N$-planet case

$$
\zeta_{i j}=\operatorname{expi}\left(\lambda_{i}-\lambda_{j}\right), \quad i, j=1,2, \ldots, N .
$$

(b) first-order closed form in terms of Jacobi elliptic functions with arguments $u_{i j}$ and modulii $k_{i j}$

$$
2 \text { am } u_{i j}=M_{i j}, \quad M_{i j}=\pi-\left(\lambda_{i}-\lambda_{j}\right), \quad k_{i j}^{2}=\frac{4 a_{i} a_{j}}{\left(a_{i}+a_{j}\right)^{2}},
$$

$a_{i}, a_{j}$ being the semi-major axes. This form involves also the elliptic quadratures, for example,

$$
\Phi_{n}(\operatorname{am} u, k, s, \alpha)=\exp (\mathrm{i} \alpha \operatorname{am} u) \int I_{s-\alpha, n-1}(u) d u
$$

with integer $n$ and $s$ and real $\alpha$. These quadratures admit a closed form representation only for integer $\alpha$. For real $\alpha$ one has to use different approximations as integration by parts (with respect to the fractional part of $s-\alpha)$, series expansion, etc.

(c) $\tau$-series form as (4.10) with

$$
\tau_{i j}=\exp i w_{i j}, \quad w_{i j}=\frac{\pi u_{i j}}{K\left(k_{i j}\right)} .
$$

Actual computation of the intermediate orbits for all major planets (Brumberg and Klioner, 1995) demonstrates the compactness of $\tau$ series with respect to $\zeta$-series.

Construction of the second-order theory by means of $\tau$-series involves the quadratures of the form

$$
I=\int f\left(u_{i j}, u_{i k}\right) d t
$$

$f$ being a trigonometric series in multiples of $w_{i j}$ and $w_{i k}$. One may use three ways to take these quadratures, i.e.

- to express integrand $f$ in terms of $M_{i j}$ and $M_{i k}$, to perform the integration and to return to variables $w_{i j}$ and $w_{i k}$;

- using the three-anomaly relation

$$
2\left(\operatorname{am} u_{i j}+\operatorname{am} u_{j k}+\operatorname{am} u_{k i}\right)=3 \pi
$$

to reduce integral (4.24) to the form

$$
I=\int g\left(u, u^{\prime}\right) d u
$$


with the relation

$$
2 \text { am } u^{\prime}=2 p \text { am } u+c, \quad c=\text { const }, \quad|p|<\frac{1}{2}
$$

and to apply the Hansen's device expressing $w^{\prime}$ in terms of $w$ and $w^{*}=p w+c$ (Brumberg, 1995);

- to integrate (4.26) by parts resulting at step $k$ in the integral of the same type with factor $p^{k}$.

Analytical integration of (4.24) by any of these ways may be rather cumbersome. In constructing a semi-analytical theory with numerical coefficients one may combine these tools with the FFT technique. Anyway, the use of elliptic function expansions permits to reduce the number of terms of the first-order theories and it remains to find the most efficient way for the higher-order theory.

\section{Elliptic Functions in Satellite Problems}

The main difficulty in analytical treatment of highly eccentric orbits is due to the slow convergence of traditional $M$-series like (2.1) for large values of eccentricity $e$. It turns out (E.Brumberg, 1992) that transformation of variables $(e, M) \rightarrow(k, u)$

$$
k=e, \quad \text { am } u=g+\frac{\pi}{2}, \quad w=\frac{\pi u}{2 K(k)}-\frac{\pi}{2}
$$

might be a possible remedy to overcome this difficulty. Classical expansions (2.1)-(2.3) are replaced therewith by rather compact expansion in multiples of the elliptic anomaly $w$

$$
\left(\frac{r}{a}\right)^{n} \exp \mathrm{i} m v=\sum_{s=-\infty}^{\infty} B_{s}^{n, m}(q) \exp \mathrm{i} s w .
$$

Most simply the coefficients of (5.2) may be computed from the recurrence relations (E.Brumberg and Fukushima, 1994).

This technique was first applied to extend for highly eccentric orbits the first-order Kaula's theory of the satellite motion in the field of the nonspherical primary (E.Brumberg et al., 1995). Later on (E.Brumberg, 1995) this technique was extended to include the third-body perturbations and was improved by using expansions (4.16) instead of (4.15).

It should be added that similar elliptic anomalies based on the transformation of the true anomaly $v$ instead of the eccentric anomaly $g$ were introduced earlier by Bond and Janin (1981) and Nacozy (1977) to improve the efficiency of the numerical integration of highly eccentric orbits. In such a way all suggestions by Gylden mentioned above are now realized. 


\section{Elliptic Functions for Nearly Intersecting Orbits}

For analytical investigation of nearly intersecting orbits (of comets, some asteroids and space probes) one may apply the elliptic function expansions in combination with the development of the disturbing function elaborated by Boda (1931), Brown and Shook (1933), Petrovskaya $(1970,1972)$ and Yuasa and Hori (1979). As suggested in the last paper one may introduce as the initial approximation for the mutual distance $\Delta$ between two bodies

$$
\Delta_{0}^{2}=r^{2}+{r^{\prime}}^{2}-2 r r^{\prime} \mu \cos \left(W-W^{\prime}\right)
$$

with the orbital longitude

$$
W=\Omega+\omega+v
$$

and the inclination factor

$$
\mu=\left(c c^{\prime}-s s^{\prime}\right)^{2}, \quad s=\sin \frac{i}{2}, \quad c=\cos \frac{i}{2} .
$$

All designations are evident. Primed quantities are referred to the disturbing body. For any integer $n$ the power expansion

$$
\Delta^{-n}=\Delta_{0}^{-n} \sum_{k=0}^{\infty} \frac{(-1)^{k}\left(\frac{n}{2}\right)_{k}}{(1)_{k}}\left(\frac{\Delta^{2}-\Delta_{0}^{2}}{\Delta_{0}^{2}}\right)^{k}
$$

converges everywhere excepting the points of the actual collision. The negative powers of $\Delta_{0}$ may be expanded in trigonometric series

$$
\Delta_{0}^{-n}=\sum_{j=0}^{\infty}\left(\Delta_{0}^{-n}\right)_{j} \cos j\left(W-W^{\prime}\right)
$$

with coefficients depending on $r$ and $r^{\prime}$ by means of hypergeometric polynomials of different arguments (Brumberg, 1995). Any function $f\left(r, r^{\prime}\right)$ occurring in (6.4) may be expanded in the symbolic form

$$
f\left(r, r^{\prime}\right)=\left(\frac{r}{a}\right)^{D}\left(\frac{r^{\prime}}{a^{\prime}}\right)^{D^{\prime}} f\left(a, a^{\prime}\right), \quad D=a \frac{\partial}{\partial a}, \quad D^{\prime}=a^{\prime} \frac{\partial}{\partial a^{\prime}} .
$$

Further expansions in terms of Jacobi nome $q$ of the disturbed body and eccentricity $e^{\prime}$ of the disturbing major planet are performed with the aid of (5.2) and (2.1) with replacing integer index $n$ by symbol $D$ or $D^{\prime}$, respectively. One returns to the classical problem of calculating Newcomb operators but in combination with elliptic anomaly expansion (5.2). 


\section{References}

Boda, K.: 1931, 'Entwicklung der Störungsfunction und ihrer Ableitungen in Reihen, welche für beliebige Exzentrizitäten und Neigungen konvergieren', Astron. Nachr., 243, 17

Bond, V.R. and Janin, G.: 1981, 'Canonical Orbital Elements in Terms of an Arbitrary Independent Variable', Celes. Mech. 23, 159

Bretagnon, P.: 1982, 'Théorie du mouvement de l'ensemble des planètes. Solution VSOP82', Astron. Astrophys. 114, 278

Brown, E.W. and Shook, C.A.: 1933, Planetary Theory, Cambridge Univ. Press

Brumberg, E. : 1992, 'Perturbed Two-Body Motion with Elliptic Functions', Proc. 25th Symposium on Celestial Mechanics (eds. H.Kinoshita and N.Nakai), 139, NAO, Tokyo

Brumberg, E.: 1995, 'Elliptic Anomaly Expansions to Construct High-Eccentricity Satellite Theory', Abstract 6a3, IAU Symposium No. 172, Paris

Brumberg, E. and Fukushima, T.: 1994, 'Expansions of Elliptic Motion Based on Elliptic Function Theory', Celes. Mech. 60, 69

Brumberg, E., Brumberg, V.A., Konrad, Th. and Soffel, M.:1995, 'Analytical Linear Perturbation Theory for Highly Eccentric Satellite Orbits', Celes. Mech. 61, 369

Brumberg, V.A.: 1995, Analytical Techniques of Celestial Mechanics, Springer

Brumberg, V.A. and Klioner, S.A.: 1995, 'Intermediate Orbit for General Planetary Theory in Elliptic Functions', Abstract A16, IAU Symposium No. 172, Paris

Chapront, J. and Chapront-Touzé, M.: 1995, 'Comparaison de la théorie du mouvement de la Lune ELP aux observations: la boite á outils', Notes sci. et techn. du BDL S050, 105

Chapront, J. and Simon, J. L.: 1988, 'Perturbations du premier ordre pour des couples de planètes', Bureau des Longitudes (unpublished)

Howland, R.A.: 1988, 'A New Approach to the Librational Solution in the Ideal Resonance Problem', Celes. Mech. 44, 209

Kinoshita, H. and Souchay, J.: 1990, 'The Theory of the Nutation for the Rigid Earth Model at the Second Order', Celes. Mech. 48, 187

Laskar, J. and Robutel, Ph.: 1995, 'Stability of the Planetary Three-Body Problem. I. Expansion of the Planetary Hamiltonian. Celes. Mech. (in press)

Nacozy, P.: 1969, 'Hansen's Method of Partial Anomalies: An Application', Astron. J. 74, 544

Nacozy, P.: 1977, 'The Intermediate Anomaly', Celes. Mech. 16, 309

Osácar, C. and Palacián, J.: 1994, 'Decomposition of Functions for Elliptic Orbits', Celes. Mech. 60, 207

Petrovskaya, M.S.: 1970, 'Expansions of the Negative Powers of Mutual Distance Between Bodies', Celes. Mech. 3, 121

Petrovskaya, M.S.: 1972, 'Expansions of the Derivatives of the Disturbing Function in Planetary Problems', Celes. Mech. 6, 328

Richardson, D.L.: 1982, 'A Third-Order Intermediate Orbit for Planetary Theory', Celes. Mech. 26, 187

Skripnichenko, V.I.: 1972, 'On the Application of Hansen's Method of Partial Anomalies to the Calculation of Perturbations in Cometary Motions', in G.A.Chebotarev, E.I.Kazimirchak-Polonskaya, and B.G.Marsden (eds.), The Motion, Evolution of Orbits, and Origin of Comets, p. 52, Reidel, Dordrecht

Williams, C.A., Van Flandern, T., and Wright, E.A.:1987, 'First Order Planetary Perturbations with Elliptic Functions', Celes. Mech. 40, 367

Yuasa, M. and Hori, G.: 1979, 'New Approach to the Planetary Theory', in R.L.Duncombe (ed.), Dynamics of the Solar System, p. 69, Reidel, Dordrecht

Zeipel, H.: 1912, 'Entwicklung der Störungsfunktion', Encyklopädie der math. Wiss. 6 (2), 557 